\title{
High Serum Uric Acid Is Associated with Tubular Damage and Kidney Inflammation in Patients with Type 2 Diabetes
}

\author{
Naiara S. Guarda, ${ }^{1}$ Yãnaí S. Bollick, ${ }^{1}$ José Antonio M. de Carvalho, ${ }^{1,2}$ Melissa O. Premaor, ${ }^{3}$ \\ Fabio V. Comim, ${ }^{3}$ and Rafael N. Moresco ${ }^{1}{ }^{1}$ \\ ${ }^{1}$ Laboratory of Clinical Biochemistry, Department of Clinical and Toxicological Analysis, Federal University of Santa Maria, \\ Santa Maria, RS, Brazil \\ ${ }^{2}$ University Hospital, Federal University of Santa Maria, Santa Maria, RS, Brazil \\ ${ }^{3}$ Department of Clinical Medicine, Federal University of Santa Maria, Santa Maria, RS, Brazil
}

Correspondence should be addressed to Rafael N. Moresco; rnmoresco@ufsm.br

Received 20 November 2018; Revised 28 January 2019; Accepted 24 February 2019; Published 11 April 2019

Academic Editor: Dennis W. T. Nilsen

Copyright (c) 2019 Naiara S. Guarda et al. This is an open access article distributed under the Creative Commons Attribution License, which permits unrestricted use, distribution, and reproduction in any medium, provided the original work is properly cited.

\begin{abstract}
Background. Uric acid presents different roles in an organism. High serum uric acid concentrations may induce inflammatory pathways and promote kidney damage through different mechanisms. Therefore, this study investigated the association among high serum uric acid concentrations, renal tubular damage, and renal inflammation assessed via estimation of urinary kidney injury molecule-1 (KIM-1) and inflammatory cytokines in patients with type 2 diabetes (T2D). Methods. Urinary concentrations of KIM-1, IL-1, IL-6, IL-10, and TNF-alpha, as well as other biochemical parameters, were assessed in 125 patients with T2D who were grouped into two groups based on the serum uric acid levels $(<6.0 \mathrm{mg} / \mathrm{dL}$ and $\geq 6.0 \mathrm{mg} / \mathrm{dL})$. Patients were also stratified according to the tertiles of serum uric acid concentrations. Results. Urinary KIM-1, IL-1, IL-6, and TNF-alpha were higher in patients with serum uric acid concentrations $\geq 6.0 \mathrm{mg} / \mathrm{dL}$. However, the differences between the groups were not statistically significant when the urinary values of KIM-1 and cytokines were normalized by the urinary creatinine concentration. Serum uric acid concentrations were significantly associated with urinary KIM-1 (values normalized by urinary creatinine concentration) and urinary TNF-alpha (absolute values and values normalized by urinary creatinine concentration), independent of the body mass index (BMI) and estimated glomerular filtration rate (eGFR). Conclusions. High serum uric acid concentrations were associated with high urinary KIM-1 levels accompanied by the increase of urinary proinflammatory cytokines in patients with T2D. However, normalization of urinary markers by urine creatinine concentration seems to influence the profile of the results.
\end{abstract}

\section{Introduction}

Hyperuricemia has emerged as a risk factor for the development of different conditions such as diabetes and cardiovascular and kidney diseases [1-4]. The association between diabetes and hyperuricemia has been extensively investigated, as such the role of uric acid in the physiopathology of diabetic kidney disease (DKD). However, the exact mechanism by which uric acid acts at the renal level is not fully understood. It is not known whether uric acid has a direct nephrotoxic effect or if it promotes some conditions leading to kidney damage. Uric acid induces hyalinosis and thickening of preglomerular arterioles, and it promotes endothelial dysfunction, glomerular hypertrophy, and activation of the renin-angiotensin system [5]. All these factors may lead to the development of kidney disease.

Increased serum uric acid is an independent risk factor for the onset and progression of microalbuminuria both in patients with diabetes and in those without diabetes $[5,6]$. Uric acid has the potential to cause damage not only at the glomerular level but also at the tubular level [7]. It is known that renal uric acid reabsorption occurs mainly in the proximal tubules [8]. Likewise, its generation occurs in tubular cells suggesting that uric acid can act directly on this portion 
of the kidney [9]. Tubular cells are important targets for the onset and progression of kidney damage. Hence, biomarkers of tubular damage can contribute substantially to improve the early detection of renal injury [10]. The kidney injury molecule-1 (KIM-1), a new marker of tubular injury, is a type I cell membrane glycoprotein expressed at low levels in healthy kidneys [11]. It has been shown that normoalbuminuric patients with type 2 diabetes (T2D) presented increased urinary levels of KIM-1 [12].

Inflammation is another pathway which plays a key role in the pathophysiology of kidney damage [13, 14]. The tubulointerstitial lesion is characterized by infiltrates of inflammatory cells with tubular atrophy and consequent interstitial fibrosis [10]. In the renal environment, inflammatory molecules such as cytokines play an important role in the development of DKD [15]. In this context, some cytokines have been highlighted as interleukin 1 (IL-1), interleukin 6 (IL-6), and tumor necrosis factor-alpha (TNF-alpha) [16]. Interestingly, uric acid is a proinflammatory factor since it can induce the expression of IL-6 and TNF-alpha through the factor nuclear kappa B (NF- $\kappa \mathrm{B})$ signaling pathway $[17,18]$. In the kidneys, these cytokines promote changes in renal and hemodynamic structures and cellular necrosis, as well as changes in the permeability of the glomerular endothelium $[15,19,20]$.

Although evidence shows the involvement of hyperuricemia and inflammation in the pathophysiology of diabetes and its chronic complications, it is still not fully understood whether elevated serum uric acid concentrations are capable of promoting increased tubular damage and renal inflammation in patients with T2D. Therefore, the aim of the present study was to investigate the association among high serum uric acid concentrations, renal tubular damage, and renal inflammation assessed via estimation of urinary KIM-1 and inflammatory cytokines in patients with T2D.

\section{Materials and Methods}

2.1. Study Population. Overall, 196 patients with T2D enrolled at the University Hospital of Santa Maria (Rio Grande do Sul, Brazil) were examined in this study. Exclusion criteria included urinary tract diseases, previous renal disease other than DKD, infectious diseases, liver diseases, acute or chronic inflammatory diseases, pregnancy, medical history of kidney transplantation or malignancy, and use of nephrotoxic drugs and anti-inflammatory and/or immunosupressive drugs. Finally, 125 patients were eligible for the study ( 85 females and 40 males). The study protocol was approved by the Institutional Ethics Committee (12303113.0.0000.5346), and written informed consent was obtained from all patients. The patients were grouped into 2 groups based on the serum uric acid levels: $<6.0 \mathrm{mg} / \mathrm{dL}$ $(n=90)$ and $\geq 6.0 \mathrm{mg} / \mathrm{dL}(n=35)$. This classification was assumed because the study population consisted mainly of female patients $(68 \%)$ and because $6.0 \mathrm{mg} / \mathrm{dL}$ is considered the upper limit of the reference range for female individuals. Furthermore, patients were also stratified according to the tertiles of serum uric acid concentrations. Clinical characteristics and medical histories of the patients were collected via a clinical and epidemiological assessment questionnaire or from the hospital's medical register. We followed in part the methods described by Stein et al. [21]. However, in the present study, a larger number of patients and new laboratory parameters were investigated.

2.2. Sample Collection and Laboratory Assays. Blood samples were collected from all patients, after an overnight fast period of at least $8 \mathrm{~h}$, by venous puncture technique into Vacutainer ${ }^{\circledR}$ tubes (BD Diagnostics, Plymouth, UK) containing EDTA, sodium fluoride plus EDTA, or no anticoagulants. The blood samples were centrifuged at $2500 \times g$ for $15 \mathrm{~min}$. Fasting glucose was measured using plasma, while serum was used to assess uric acid, total cholesterol, HDL cholesterol, LDL cholesterol, and triglycerides. These measurements were performed using standard methods on the Dimension RxL Max ${ }^{\circledR}$ automated analyzer (Siemens Healthcare Diagnostics Inc., Malvern, Pennsylvania, USA). The EDTA containing whole blood samples were used to measure glycated hemoglobin $\left(\mathrm{HbA}_{1 \mathrm{c}}\right)$ by use of a chromatographic method on the D-10 ${ }^{\circledR}$ analyzer (Bio-Rad, California, USA). First-morning urine samples were obtained from the patients and centrifuged at $1000 \times g$ for $5 \mathrm{~min}$. The supernatants were used to measure albumin, creatinine, KIM-1, IL-1, IL-6, IL10 , and TNF-alpha. Urinary albumin was quantified by use of an immunoturbidimetric assay on the Dimension RxL $\mathrm{Max}^{\circledR}$ automated analyzer, and the results were expressed as the albumin/creatinine ratio (ACR) as a tool to match the levels of albumin in accordance with the concentration of urine [22]. Urinary KIM-1, IL-1, IL-6, IL-10, and TNFalpha were measured using commercial ELISA kits (R\&D Systems Inc., Minneapolis, Minnesota, USA). All tests were performed according to the instructions recommended by the manufacturer. The estimated glomerular filtration rate (eGFR) was calculated using the creatinine equation obtained from the Chronic Kidney Disease Epidemiology Collaboration (CKD-EPI) [23].

2.3. Statistical Analysis. The variables were tested for normality using the D'Agostino-Pearson test. The parametric variables are presented as mean \pm standard deviation (SD), and the nonparametric variables are presented as the median and interquartile range (IQR). Statistical differences between the groups were analyzed using Student's $t$ test or the Mann-Whitney test. The categorical data are expressed as percentages, and differences between the groups were compared using the Chi-square test. Additionally, multiple regression analysis was performed to investigate the influence of some variables on urinary KIM-1 levels. Two-tailed $P$ values $<0.05$ were considered statistically significant. All results were analyzed using GraphPad Prism ${ }^{\circledR}$ version 6.00 (GraphPad Software, La Jolla, California, USA) and Statistica ${ }^{\circledR}$ version 9.1 (StatSoft Inc., Tulsa, Oklahoma, USA).

\section{Results}

The baseline characteristics of the participants included in the study are shown in Table 1. No differences in age, the proportion of smokers, and the proportion of patients with 
TABLE 1: Baseline characteristics and biochemical parameters of the study participants $(n=125)$ stratified according to serum uric acid concentrations.

\begin{tabular}{|c|c|c|c|}
\hline & Serum uric acid $<6.0 \mathrm{mg} / \mathrm{dL}$ & Serum uric acid $\geq 6.0 \mathrm{mg} / \mathrm{dL}$ & $P$ value \\
\hline Age (y) & $60(50-68)$ & $59(46-68)$ & 0.644 \\
\hline Male (\%) & 23.3 & 54.3 & 0.001 \\
\hline BMI $\left(\mathrm{kg} / \mathrm{m}^{2}\right)$ & $30.2(26.8-36.0)$ & $34.70(28.6-40.7)$ & 0.038 \\
\hline Hypertension (\%) & 69.7 & 88.6 & 0.029 \\
\hline Smokers (\%) & 5.9 & 16.1 & 0.082 \\
\hline Diabetes duration (years) & $12.0(8.0-18.0)$ & $10.0(5.0-21.2)$ & 0.705 \\
\hline Hypoglycemic agents (\%) & 95.6 & 91.4 & 0.359 \\
\hline ACE inhibitors (\%) & 31.1 & 61.8 & 0.002 \\
\hline Other antihypertensive agents (\%) & 67.0 & 88.5 & 0.015 \\
\hline Statin use $(\%)$ & 74.7 & 62.9 & 0.195 \\
\hline Alopurinol use (\%) & 5.7 & 6.2 & 1.000 \\
\hline Insulin use $(\%)$ & 35.2 & 20.0 & 0.132 \\
\hline Serum uric acid (mg/dL) & $4.5 \pm 0.8$ & $7.0 \pm 0.8$ & $<0.001$ \\
\hline Fasting glucose $(\mathrm{mmol} / \mathrm{L})$ & $6.5(5.5-8.8)$ & $6.6(5.8-8.4)$ & 0.870 \\
\hline $\mathrm{HbA}_{1 \mathrm{c}}(\%)$ & $6.9(6.0-8.5)$ & $6.4(5.9-8.6)$ & 0.878 \\
\hline $\mathrm{HbA}_{1 \mathrm{c}}(\mathrm{mmol} / \mathrm{mol})$ & $52(42-69)$ & $46(41-70)$ & 0.878 \\
\hline Total cholesterol (mmol/L) & $4.6(4.2-4.9)$ & $4.1(3.5-5.2)$ & 0.036 \\
\hline LDL cholesterol (mmol/L) & $2.6 \pm 0.6$ & $2.4 \pm 0.8$ & 0.202 \\
\hline HDL cholesterol (mmol/L) & $1.2(1.0-1.5)$ & $1.0(0.9-1.3)$ & 0.011 \\
\hline $\mathrm{eGFR}\left(\mathrm{mL} / \mathrm{min} / 1.73 \mathrm{~m}^{2}\right)$ & $83.9 \pm 21.3$ & $73.5 \pm 27.7$ & 0.064 \\
\hline Urinary ACR (mg/g creat) & $7.6(5.2-14.3)$ & $8.0(5.8-35.3)$ & 0.416 \\
\hline
\end{tabular}

Data are expressed as percentages, mean \pm standard deviation, or median and interquartile range. ACE inhibitors: angiotensin-converting enzyme inhibitors ACR: albumin/creatinine ratio; BMI: body mass index; eGFR: estimated glomerular filtration; $\mathrm{HbA}_{1 \mathrm{c}}$ : glycated hemoglobin.

T2D were observed between the groups. However, significant differences were observed concerning gender, BMI, and proportion of hypertensive patients. Plasma fasting glucose, blood $\mathrm{HbA}_{1 \mathrm{c}}$, serum LDL cholesterol, and eGFR levels were not statistically different between the groups. However, serum total cholesterol levels were higher, and HDL cholesterol levels were lower in the group with uric acid $\geq 6.0 \mathrm{mg} / \mathrm{dL}$, when compared with the group with serum uric acid $<6.0 \mathrm{mg} / \mathrm{dL}$.

The urinary KIM-1 levels were significantly higher in patients with high serum uric acid when compared with those with low serum uric acid $(122(80-162)$ versus 80 $(58-124) \mathrm{pg} / \mathrm{mL}, P=0.0309)$, as shown in Figure 1(a). However, the difference between the groups was not significant when the urinary values of KIM-1 were normalized by the urinary creatinine concentration (Figure 1(b)). The ACR levels observed in the patients did not differ statistically between the groups (Table 1). Furthermore, urinary concentrations of IL-1, IL-6, and TNF-alpha were significantly higher in patients with high serum uric acid when compared with those with low serum uric acid, as shown in Figure 2. Although a decreasing trend in the urinary IL-10 in the group of patients with high serum uric acid was observed, the difference between the groups was not statistically significant. Interestingly, the differences were not statistically significant when urinary cytokine concentrations were normalized by the urinary creatinine concentration (Figure 3).
Additionally, the patients were stratified according to the tertiles of serum uric acid concentrations $\left(1^{\text {st }}\right.$ tertile: $2.7-4.5 \mathrm{mg} / \mathrm{dL}, 2^{\text {nd }}$ tertile: $4.6-5.7 \mathrm{mg} / \mathrm{dL}$, and $3^{\text {rd }}$ tertile: $5.8-9.0 \mathrm{mg} / \mathrm{dL}$ ). Based on this stratification, there were differences between the groups only for KIM-1 (absolute values and normalized by urinary creatinine concentration), as shown in Table 2.

Although differences in urinary cytokines were not statistically significant, there was a trend toward elevation of IL-1, IL-6, and TNF-alpha in the third tertile. The multiple regression analysis was conducted using two models that included urinary KIM-1 and urinary cytokines, as well as other variables. Serum uric acid concentrations were significantly associated with urinary KIM-1 (values normalized by urinary creatinine concentration) and urinary TNF-alpha (absolute values and values normalized by urinary creatinine concentration), independent of the BMI and eGFR (Table 3).

\section{Discussion}

The associations among serum uric acid levels, tubular damage, and renal inflammation were investigated in the present study. Interestingly, we observed the cooccurrence of high serum uric acid with high urinary KIM-1, IL-1, IL-6, and TNF-alpha in patients with T2D. However, these differences were no longer significant when the values were corrected by the urinary creatinine concentration. This was an intriguing 


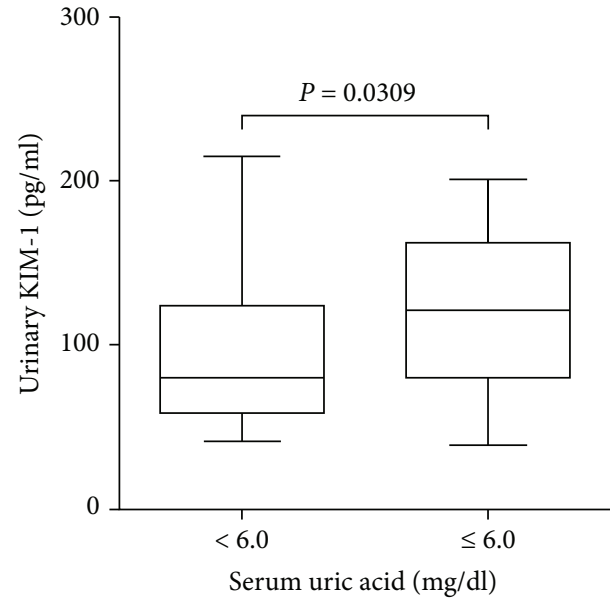

(a)

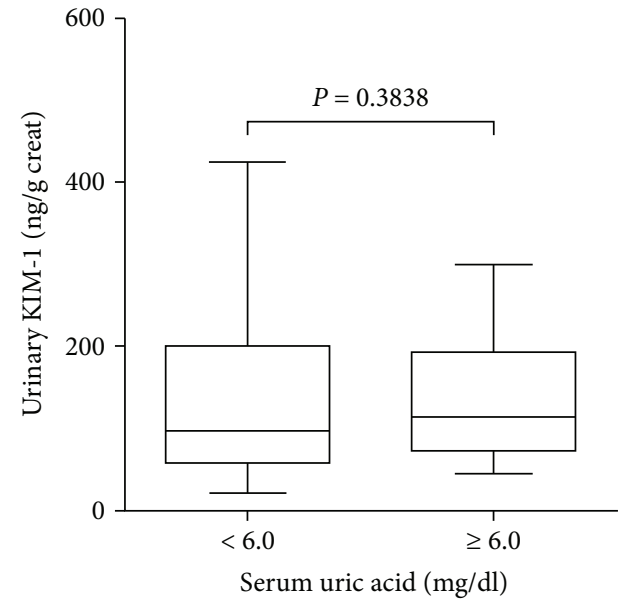

(b)

FIGURE 1: Box-and-whisker plots showing the urinary values of KIM-1 reported as (a) absolute values and (b) values normalized by urinary creatinine concentration. Subjects $(n=125)$ were stratified based on serum uric acid levels $<6.0 \mathrm{mg} / \mathrm{dL}$ and $\geq 6.0 \mathrm{mg} / \mathrm{dL}$. The box contained $50 \%$ of all values (from the $25^{\text {th }}$ to $75^{\text {th }}$ percentile) and was divided by the horizontal bar representing the median value $\left(50^{\text {th }}\right.$ percentile).

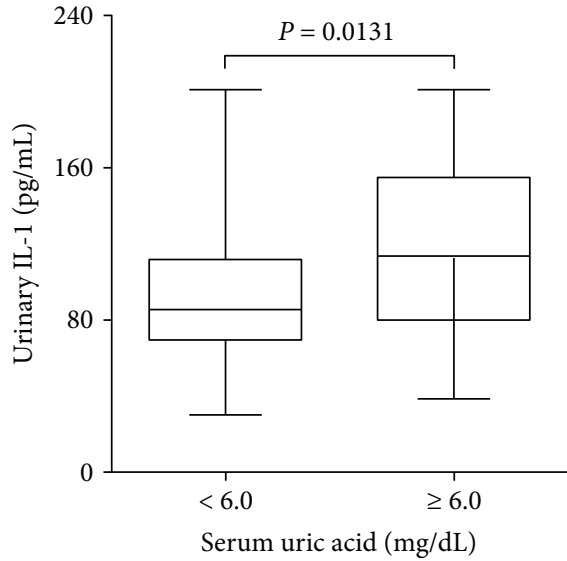

(a)

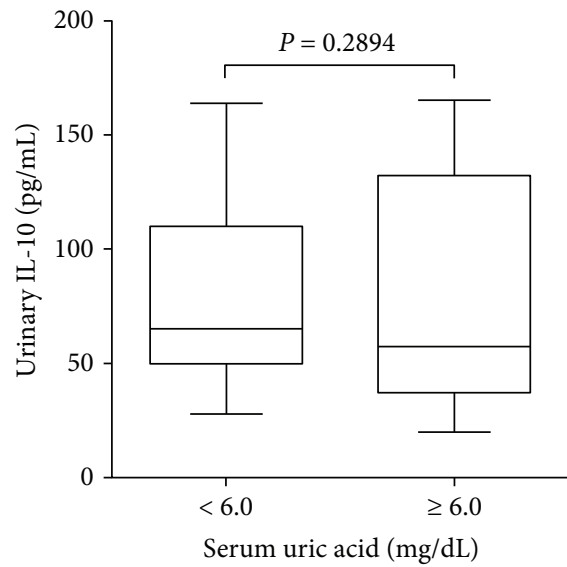

(c)

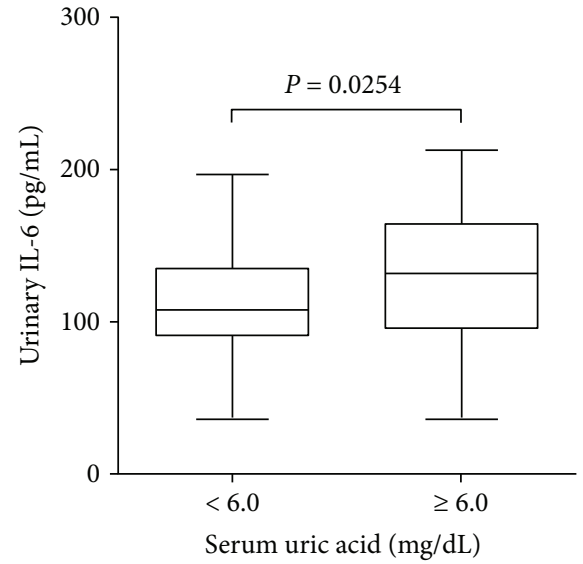

(b)

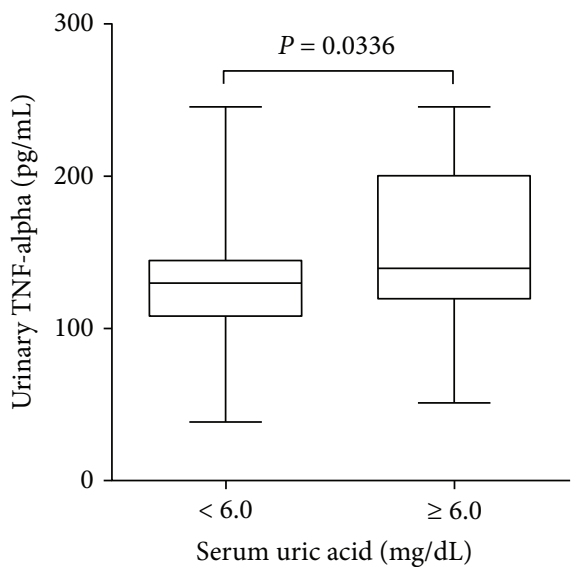

(d)

FIgure 2: Box-and-whisker plots are showing the urinary concentrations of IL-1 (a), IL-6 (b), IL-10 (c), and TNF-alpha (d) reported as absolute values. Subjects $(n=125)$ were stratified based on serum uric acid levels $<6.0 \mathrm{mg} / \mathrm{dL}$ and $\geq 6.0 \mathrm{mg} / \mathrm{dL}$. The box contained $50 \%$ of all values (from the $25^{\text {th }}$ to $75^{\text {th }}$ percentile) and was divided by the horizontal bar representing the median value $\left(50^{\text {th }}\right.$ percentile). 


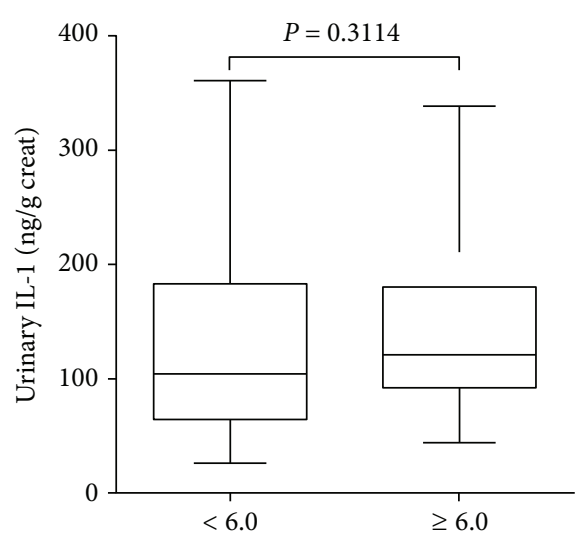

(a)

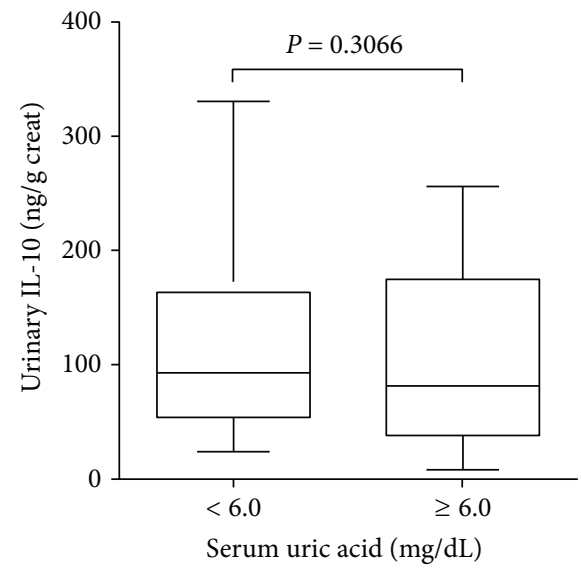

(c)

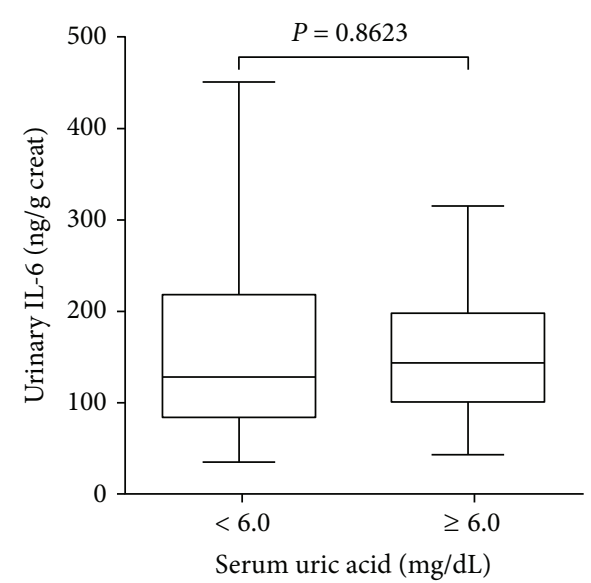

(b)

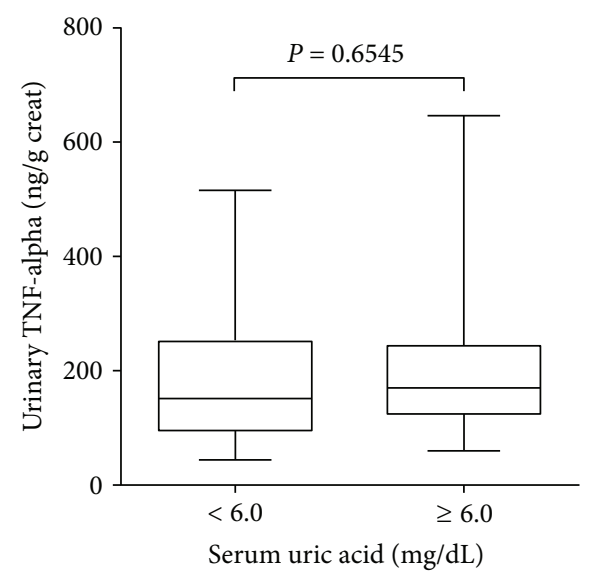

(d)

FIGURE 3: Box-and-whisker plots are showing the urinary concentrations of IL-1 (a), IL-6 (b), IL-10 (c), and TNF-alpha (d) reported as values normalized by urinary creatinine concentration. Subjects $(n=125)$ were stratified based on serum uric acid levels $<6.0 \mathrm{mg} / \mathrm{dL}$ and $\geq 6.0 \mathrm{mg} / \mathrm{dL}$. The box contained $50 \%$ of all values (from the $25^{\text {th }}$ to $75^{\text {th }}$ percentile) and was divided by the horizontal bar representing the median value $\left(50^{\text {th }}\right.$ percentile).

TABLE 2: Biochemical parameters of the study participants $(n=125)$ stratified according to tertiles of serum uric acid concentration.

\begin{tabular}{|c|c|c|c|c|}
\hline & $1^{\text {st }}$ tertile & $2^{\text {nd }}$ tertile & $3^{\text {rd }}$ tertile & $P$ value \\
\hline Urinary KIM-1 (pg/mL) & $96(56-144)$ & $77(58-95)$ & $97(79-159)$ & 0.039 \\
\hline Urinary KIM-1 (ng/g creat) & $121(76-265)$ & $76(48-107)$ & $125(88-200)$ & 0.009 \\
\hline Urinary IL-1 (pg/mL) & $93(73-131)$ & $82(66-100)$ & $100(78-143)$ & 0.065 \\
\hline Urinary IL-1 (ng/g creat) & $108(67-192)$ & $88(53-156)$ & $123(94-187)$ & 0.057 \\
\hline Urinary IL-6 (pg/mL) & $115(91-144)$ & $99(92-124)$ & $121(96-155)$ & 0.098 \\
\hline Urinary IL-6 (ng/g creat) & $129(88-227)$ & $124(70-184)$ & $171(103-213)$ & 0.141 \\
\hline Urinary IL-10 (pg/mL) & $62(46-90)$ & $69(51-135)$ & $64(39-130)$ & 0.379 \\
\hline Urinary IL-10 (ng/g creat) & $76(47-152)$ & $93(54-164)$ & $87(40-178)$ & 0.846 \\
\hline Urinary TNF-alpha (pg/mL) & $137(110-161)$ & $128(110-139)$ & $132(106-187)$ & 0.267 \\
\hline Urinary TNF-alpha (ng/g creat) & $142(104-267)$ & $149(83-189)$ & $176(124-251)$ & 0.272 \\
\hline
\end{tabular}

Data are expressed as median and interquartile ranges. Parameters are reported as absolute values and as values normalized by urinary creatinine concentration.

finding since there is no definite consensus on the need for normalization of urinary KIM-1 and cytokines by the concentration of creatinine in the urine.

Adjustment of results by urinary creatinine is usually performed to compensate for variations in urine concentrations, but the urinary excretion of creatinine can vary for different reasons. Most evidence seems to support the use of tubular biomarkers such as KIM-1 expressed as values corrected for urinary creatinine [24-27]. However, some studies expressed the results of KIM-1 only as values adjusted by urinary 
TABLE 3: Multiple regression analysis of serum uric acid as a dependent variable adjusting for some clinical and laboratory variables of the study population $(n=125)$.

\begin{tabular}{|c|c|c|c|c|}
\hline & $B$ & $\mathrm{SE}_{B}$ & $t$ & $P$ value \\
\hline \multicolumn{5}{|l|}{ Model 1} \\
\hline Male $(\%)$ & -0.546 & 0.357 & -1.527 & 0.133 \\
\hline BMI $\left(\mathrm{kg} / \mathrm{m}^{2}\right)$ & 0.061 & 0.017 & 3.486 & 0.001 \\
\hline Hypertension (\%) & 1.806 & 1.210 & 1.492 & 0.142 \\
\hline ACE inhibitors (\%) & 0.125 & 0.357 & 0.349 & 0.729 \\
\hline Other antihypertensive agents (\%) & -1.142 & 1.176 & -0.971 & 0.336 \\
\hline Total cholesterol (mmol/L) & -0.004 & 0.203 & -0.021 & 0.984 \\
\hline $\mathrm{eGFR}\left(\mathrm{mL} / \mathrm{min} / 1.73 \mathrm{~m}^{2}\right)$ & -0.013 & 0.007 & -1.710 & 0.093 \\
\hline Urinary ACR (mg/g creat) & 0.005 & 0.002 & 1.977 & 0.053 \\
\hline Urinary KIM-1 (pg/mL) & -0.001 & 0.004 & -0.176 & 0.861 \\
\hline Urinary KIM-1 (ng/g creat) & -0.004 & 0.001 & -3.090 & 0.003 \\
\hline \multicolumn{5}{|l|}{ Model 2} \\
\hline Male (\%) & -0.304 & 0.322 & -0.944 & 0.349 \\
\hline BMI $\left(\mathrm{kg} / \mathrm{m}^{2}\right)$ & 0.055 & 0.017 & 3.341 & 0.001 \\
\hline Hypertension (\%) & 1.863 & 1.226 & 1.519 & 0.134 \\
\hline ACE inhibitors (\%) & 0.348 & 0.318 & 1.095 & 0.278 \\
\hline Other antihypertensive agents (\%) & -1.234 & 1.201 & -1.027 & 0.308 \\
\hline Total cholesterol (mmol/L) & -0.175 & 0.197 & -0.890 & 0.377 \\
\hline $\mathrm{eGFR}\left(\mathrm{mL} / \mathrm{min} / 1.73 \mathrm{~m}^{2}\right)$ & -0.018 & 0.006 & -2.728 & 0.008 \\
\hline Urinary ACR (mg/g creat) & 0.003 & 0.002 & 1.328 & 0.189 \\
\hline Urinary IL-1 (pg/mL) & -0.025 & 0.020 & -1.254 & 0.215 \\
\hline Urinary IL-1 (ng/g creat) & 0.006 & 0.013 & 0.537 & 0.593 \\
\hline Urinary IL-6 (pg/mL) & -0.021 & 0.022 & -0.991 & 0.326 \\
\hline Urinary IL-6 (ng/g creat) & 0.017 & 0.011 & 1.545 & 0.128 \\
\hline Urinary IL-10 (pg/mL) & 0.001 & 0.006 & 0.043 & 0.966 \\
\hline Urinary IL-10 (ng/g creat) & 0.002 & 0.004 & 0.372 & 0.711 \\
\hline Urinary TNF-alpha (pg/mL) & 0.046 & 0.017 & 2.673 & 0.009 \\
\hline Urinary TNF-alpha (ng/g creat) & -0.024 & 0.010 & -2.464 & 0.017 \\
\hline
\end{tabular}

Regression coefficients $(B)$, standard error of $B\left(\mathrm{SE}_{B}\right)$, and $t$ statistic with corresponding $P$ value. Analyses were performed considering urinary concentrations of KIM-1 and cytokines as absolute values and normalized by urinary creatinine.

creatinine and not as absolute values. Thus, it is not possible to conclude on the influence of the adjustment of the KIM-1 results by urinary creatinine on the performance of the marker in these clinical conditions. Conti et al. [28] investigated the effect of urinary creatinine on urinary cystatin as a model of a tubular marker, and urinary cystatin was superior to the urinary cystatin/creatinine ratio to evaluate the extent of renal tubular damage. Therefore, it was recommended to avoid the adjustment of tubular markers to urinary creatinine, especially in patients with acute or even moderate chronic renal failure. Interestingly, a study from our group reported that urinary KIM-1 had a higher ability to discriminate incipient diabetic kidney disease than the urinary KIM-1/creatinine ratio [29]. In contrast, another study showed that both urinary cystatin C and KIM-1 concentrations were positively correlated with urinary creatinine, indicating a rationale for urinary creatinine adjustment [30].
Some studies have investigated the role of uric acid in causing kidney damage [3-6, 31]. The uric acid at high levels has the potential to induce renal injury through different mechanisms such as renal vasoconstriction mediated by endothelial dysfunction, inflammation, activation of the renin-angiotensin system, and afferent renal arteriolopathy [31-35]. Uric acid may promote the phenotypic transition of renal cells even at physiological concentrations [7]. Likewise, high concentrations of uric acid may cause tubulointerstitial fibrosis [7]. Furthermore, a recent study from our group [21] reported the cooccurrence of high serum uric acid with high urinary 8-hydroxydeoxyguanosine (8-OHdG), a marker of nucleoside oxidation, in patients with T2D. We speculate that serum uric acid at high concentrations combined with other prooxidant changes observed in diabetes appears to potentiate the formation of $8-\mathrm{OHdG}$ in T2D patients [21].

In the present study, we investigated the influence of serum uric acid on tubular damage in patients with T2D and observed that it occurred most strongly in patients with T2D with higher serum uric acid concentrations. Interestingly, a previous study reported the occurrence of high concentrations of urinary KIM-1 in adolescents with hyperuricemia compared to healthy adolescents [36]. Another finding of the present study was that similar ACR levels were found in both groups of patients. It is known that tubular damage may precede and even occur independently of glomerular dysfunction. For this reason, the assessment of markers of early tubular renal damage such as KIM-1 becomes a valuable tool [37-39]. Following tubular damage, the injured kidney cells have deranged expression and secretion of KIM-1, and it is a potential marker for monitoring the degree of tubular epithelial cell injury [39]. Although the exact mechanism of the released soluble KIM-1 remains unknown, it has been suggested that KIM-1 can participate in the injury process since it plays an important role in the proliferation and regeneration processes in proximal tubules [11, 39, 40].

Inflammation has an important role in the pathophysiology of diabetes mellitus, and it contributes significantly to the development of renal disease [41, 42]. Interestingly, uric acid appears to play a key role both in inflammation and in kidney damage $[32,43]$. Furthermore, uric acid may promote renal inflammation via the NF- $\kappa \mathrm{B}$ signaling pathway [44]. The production of interleukin- $1 \beta$ resulting from the induction of the NLRP3 pathway in renal mesangial cells through the action of uric acid appears to be another important mechanism for the development of kidney damage [45]. We had previously reported an increase of urinary IL-6 combined with a decrease in urinary IL-10 in patients with DKD [46]. However, in the present study, we investigated the influence of serum uric acid on renal inflammation in patients with T2D and observed that it occurred most evidently in patients with T2D with higher serum uric acid concentrations. Thus, we speculate that serum uric acid at high concentrations, when combined with other metabolic and prooxidant changes observed in diabetes, seems to enhance the proinflammatory cytokines involving IL-1, IL-6, and TNF-alpha in T2D patients. However, differences were not statistically significant 
when urinary cytokine concentrations were normalized by the urinary creatinine concentration. Thus, it is important to reflect on the need to correct the results of urinary cytokines by the concentration of creatinine in the urine, similar to what happens with tubular markers such as KIM-1. It is well established that proinflammatory cytokines may promote renal effects through several pathways. IL-1 and IL- 6 can promote hypercellularity and interstitial fibrosis in the renal tubules $[47,48]$, while TNF-alpha has cytotoxic action on mesangial and epithelial glomerular cells [15, 49].

Unfortunately, this study has some limitations. Firstly, the number of patients enrolled was relatively small. For this reason, some of the associations reported in the present study were not very strong, which may have been influenced by this relatively small number of investigated patients. Secondly, the potential contribution of systemic cytokine concentrations to their urinary levels was not known since serum levels of cytokines were not measured. Thirdly, first-morning urine samples were used to quantify urinary biomarkers rather than timed urine specimens, which would allow a more accurate estimate of the excretion rate. Furthermore, it was not possible to investigate in more depth the effects of the use of certain drugs that may affect serum uric acid on urinary markers measured in this study.

\section{Conclusion}

High concentrations of serum uric acid were associated with tubular damage accompanied by the increase of urinary proinflammatory cytokines in patients with T2D. However, normalization of urinary markers by urine creatinine concentration seems to influence the profile of the results. Therefore, a standardization for the expression of the results of markers like KIM-1 and cytokines in urine samples is required. It seems reasonable to consider the need to correct the results of tubular markers in the urine by urinary creatinine concentration due to a wide variety of factors, which may affect the dilution of urine. Meanwhile, presenting the results of tubular markers in urine such as KIM-1 expressed both as absolute values and values normalized by urinary creatinine seems to be the best strategy. Serum uric acid concentrations were associated with urinary KIM-1 and urinary TNF-alpha, independent of the BMI and eGFR. We hypothesize that serum uric acid at high concentrations, combined with other changes observed in T2D, may contribute to the enhancement of kidney inflammation and tubular damage. However, further longitudinal studies are required to address the real impact of serum uric acid on the development of tubular damage and kidney inflammation in patients with T2D.

\section{Data Availability}

The data used to support the findings of this study are included within the article.

\section{Disclosure}

The funding organization(s) played no role in the study design; in the collection, analysis, and interpretation of data; in the writing of the report; or in the decision to submit the report for publication.

\section{Conflicts of Interest}

The authors declare that they have no conflicts of interest.

\section{Authors' Contributions}

All the authors have accepted responsibility for the entire content of this submitted manuscript and approved submission.

\section{Acknowledgments}

The authors thank Bioclin/Quibasa (Belo Horizonte, Brazil) for providing biochemical reagents. This study was supported by the Conselho Nacional de Desenvolvimento Científico e Tecnológico (CNPq, Brazil, number 428321/2016-0) and Coordenação de Aperfeiçoamento de Pessoal de Nível Superior (CAPES, Brazil, finance code 001). R.N. Moresco and M.O. Premaor are recipients of research productivity scholarships from the $\mathrm{CNPq} /$ Brazil.

\section{References}

[1] J. F. Baker, E. Krishnan, L. Chen, and H. R. Schumacher, "Serum uric acid and cardiovascular disease: recent developments, and where do they leave us?," The American Journal of Medicine, vol. 118, no. 8, pp. 816-826, 2005.

[2] A. Dehghan, M. van Hoek, E. J. G. Sijbrands, A. Hofman, and J. C. M. Witteman, "High serum uric acid as a novel risk factor for type 2 diabetes," Diabetes Care, vol. 31, no. 2, pp. 361-362, 2008.

[3] D. Yan, Y. Tu, F. Jiang et al., "Uric acid is independently associated with diabetic kidney disease: a cross-sectional study in a Chinese population," PLoS One, vol. 10, no. 6, article e0129797, 2015.

[4] V. Bartáková, K. Kuricová, L. Pácal et al., "Hyperuricemia contributes to the faster progression of diabetic kidney disease in type 2 diabetes mellitus," Journal of Diabetes and its Complications, vol. 30, no. 7, pp. 1300-1307, 2016.

[5] H. Y. Chang, P. H. Lee, C. C. Lei et al., "Hyperuricemia is an independent risk factor for new onset micro-albuminuria in a middle-aged and elderly population: a prospective cohort study in Taiwan," PLoS One, vol. 8, no. 4, article e61450, 2013.

[6] Y. Hayashino, S. Okamura, S. Tsujii, and H. Ishii, "Association of serum uric acid levels with the risk of development or progression of albuminuria among Japanese patients with type 2 diabetes: a prospective cohort study [Diabetes Distress and Care Registry at Tenri (DDCRT 10)]," Acta Diabetologica, vol. 53, no. 4, pp. 599-607, 2016.

[7] E. S. Ryu, M. J. Kim, H. S. Shin et al., "Uric acid-induced phenotypic transition of renal tubular cells as a novel mechanism of chronic kidney disease," American Journal of PhysiologyRenal Physiology, vol. 304, no. 5, pp. F471-F480, 2013.

[8] B. Shekarriz and M. L. Stoller, "Uric acid nephrolithiasis: current concepts and controversies," The Journal of Urology, vol. 168, no. 4, Part 1, pp. 1307-1314, 2002.

[9] P. Cirillo, M. S. Gersch, W. Mu et al., "Ketohexokinasedependent metabolism of fructose induces proinflammatory 
mediators in proximal tubular cells," Journal of the American Society of Nephrology, vol. 20, no. 3, pp. 545-553, 2009.

[10] S. M. Bagshaw and R. Bellomo, "Early diagnosis of acute kidney injury," Current Opinion in Critical Care, vol. 13, no. 6, pp. 638-644, 2007.

[11] W. K. Han, V. Bailly, R. Abichandani, R. Thadhani, and J. V. Bonventre, "Kidney injury molecule-1 (KIM-1): a novel biomarker for human renal proximal tubule injury," Kidney International, vol. 62, no. 1, pp. 237-244, 2002.

[12] J. A. M. de Carvalho, E. Tatsch, B. S. Hausen et al., "Urinary kidney injury molecule-1 and neutrophil gelatinase-associated lipocalin as indicators of tubular damage in normoalbuminuric patients with type 2 diabetes," Clinical Biochemistry, vol. 49, no. 3, pp. 232-236, 2016.

[13] J. J. Friedewald and H. Rabb, "Inflammatory cells in ischemic acute renal failure," Kidney International, vol. 66, no. 2, pp. 486-491, 2004.

[14] M. L. Kielar, R. John, M. Bennett et al., "Maladaptive role of IL-6 in ischemic acute renal failure," Journal of the American Society of Nephrology, vol. 16, no. 11, pp. 3315-3325, 2005.

[15] J. F. Navarro-González and C. Mora-Fernández, "The role of inflammatory cytokines in diabetic nephropathy," Journal of the American Society of Nephrology, vol. 19, no. 3, pp. 433-442, 2008.

[16] K. Alexandraki, C. Piperi, C. Kalofoutis, J. Singh, A. Alaveras, and A. Kalofoutis, "Inflammatory process in type 2 diabetes: the role of cytokines," Annals of the New York Academy of Sciences, vol. 1084, no. 1, pp. 89-117, 2006.

[17] M. Suganuma, S. Okabe, M. Kurusu et al., "Discrete roles of cytokines, TNF- $\alpha$, IL-1, IL-6 in tumor promotion and cell transformation," International Journal of Oncology, vol. 20, pp. 131-136, 2002.

[18] C. C. Huang, B. S. Lou, F. L. Hsu, and C. C. Hou, "Use of urinary metabolomics to evaluate the effect of hyperuricemia on the kidney," Food and Chemical Toxicology, vol. 74, pp. 35-44, 2014.

[19] J. Pfeilschifter, W. Pignat, K. Vosbeck, and F. Märki, "Interleukin 1 and tumor necrosis factor synergistically stimulate prostaglandin synthesis and phospholipase A2 release from rat renal mesangial cells," Biochemical and Biophysical Research Communications, vol. 159, no. 2, pp. 385-394, 1989.

[20] D. L. Coleman and C. Ruef, "Interleukin 6: an autocrine regulator of mesangial cell growth," Kidney International, vol. 41, no. 3, pp. 604-606, 1992.

[21] C. S. Stein, J. A. M. de Carvalho, M. M. M. F. Duarte et al., "High serum uric acid is associated with oxidation of nucleosides in patients with type 2 diabetes," Mutation Research/Fundamental and Molecular Mechanisms of Mutagenesis, vol. 811, pp. 27-30, 2018.

[22] D. J. Newman, M. J. Pugia, J. A. Lott, J. F. Wallace, and A. M. Hiar, "Urinary protein and albumin excretion corrected by creatinine and specific gravity," Clinica Chimica Acta, vol. 294, no. 1-2, pp. 139-155, 2000.

[23] A. S. Levey, L. A. Stevens, C. H. Schmid et al., "A new equation to estimate glomerular filtration rate," Annals of Internal Medicine, vol. 150, no. 9, pp. 604-612, 2009.

[24] V. R. Curovic, T. W. Hansen, M. K. Eickhoff et al., "Urinary tubular biomarkers as predictors of kidney function decline, cardiovascular events and mortality in microalbuminuric type 2 diabetic patients," Acta Diabetologica, vol. 55, no. 11, pp. 1143-1150, 2018.
[25] A. C. Carlsson, A. Larsson, J. Helmersson-Karlqvist et al., "Urinary kidney injury molecule 1 and incidence of heart failure in elderly men," European Journal of Heart Failure, vol. 15, no. 4, pp. 441-446, 2013.

[26] T. H. Driver, R. Katz, J. H. Ix et al., "Urinary kidney injury molecule 1 (KIM-1) and interleukin 18 (IL-18) as risk markers for heart failure in older adults: the Health, Aging, and Body Composition (Health ABC) Study," American Journal of Kidney Diseases, vol. 64, no. 1, pp. 49-56, 2014.

[27] K. Damman, S. Masson, H. L. Hillege et al., "Clinical outcome of renal tubular damage in chronic heart failure," European Heart Journal, vol. 32, no. 21, pp. 2705-2712, 2011.

[28] M. Conti, S. Moutereau, L. Esmilaire et al., "Should kidney tubular markers be adjusted for urine creatinine? The example of urinary cystatin C," Clinical Chemistry and Laboratory Medicine, vol. 47, no. 12, pp. 1553-1556, 2009.

[29] J. A. M. De Carvalho, E. Tatsch, B. S. Hausen et al., "Evaluation of the diagnostic characteristics of urinary kidney injury molecule 1 (uKIM-1) and uKIM-1/creatinine ratio in the assessment of incipient diabetic kidney disease," Clinical Chemistry and Laboratory Medicine (CCLM), vol. 53, no. 2, pp. e51e54, 2015.

[30] J. Helmersson-Karlqvist, J. Ärnlöv, A. C. Carlsson, L. Lind, and A. Larsson, "Urinary KIM-1, but not urinary cystatin C, should be corrected for urinary creatinine," Clinical Biochemistry, vol. 49, no. 15, pp. 1164-1166, 2016.

[31] P. Hovind, P. Rossing, R. J. Johnson, and H. H. Parving, "Serum uric acid as a new player in the development of diabetic nephropathy," Journal of Renal Nutrition, vol. 21, no. 1, pp. 124-127, 2011.

[32] G. Zoppini, G. Targher, M. Chonchol et al., "Serum uric acid levels and incident chronic kidney disease in patients with type 2 diabetes and preserved kidney function," Diabetes Care, vol. 35, no. 1, pp. 99-104, 2012.

[33] F. Preitner, A. Pimentel, S. Metref et al., "No development of hypertension in the hyperuricemic liver-Glut9 knockout mouse," Kidney International, vol. 87, no. 5, pp. 940-947, 2015.

[34] D.-H. Kang, T. Nakagawa, L. Feng et al., "A role for uric acid in the progression of renal disease," Journal of the American Society of Nephrology, vol. 13, no. 12, pp. 2888-2897, 2015.

[35] L. G. Sánchez-Lozada, E. Tapia, C. Avila-Casado et al., "Mild hyperuricemia induces glomerular hypertension in normal rats," American Journal of Physiology-Renal Physiology, vol. 283, no. 5, pp. F1105-F1110, 2002.

[36] J. Tomczak, A. Wasilewska, and R. Milewski, "Urine NGAL and KIM-1 in children and adolescents with hyperuricemia," Pediatric Nephrology, vol. 28, no. 9, pp. 1863-1869, 2013.

[37] S. E. Nielsen, H. Reinhard, D. Zdunek et al., "Tubular markers are associated with decline in kidney function in proteinuric type 2 diabetic patients," Diabetes Research and Clinical Practice, vol. 97, no. 1, pp. 71-76, 2012.

[38] O. Liangos, M. C. Perianayagam, V. S. Vaidya et al., "Urinary $\mathrm{N}$-acetyl- $\beta$-(D) glucosaminidase activity and kidney injury molecule-1 level are associated with adverse outcomes in acute renal failure," Journal of the American Society of Nephrology, vol. 18, no. 3, pp. 904-912, 2007.

[39] R. N. Moresco, G. V. Bochi, C. S. Stein, J. A. M. de Carvalho, B. M. Cembranel, and Y. S. Bollick, "Urinary kidney injury molecule-1 in renal disease," Clinica Chimica Acta, vol. 487, pp. 15-21, 2018. 
[40] T. Ichimura, C. R. Brooks, and J. V. Bonventre, "Kim-1/Tim-1 and immune cells: shifting sands," Kidney International, vol. 81, no. 9, pp. 809-811, 2012.

[41] M. D. Williams and J. L. Nadler, "Inflammatory mechanisms of diabetic complications," Current Diabetes Reports, vol. 7, no. 3, pp. 242-248, 2007.

[42] J. F. Navarro and C. Mora, "Role of inflammation in diabetic complications," Nephrology Dialysis Transplantation, vol. 20, no. 12, pp. 2601-2604, 2005.

[43] M. B. Duran-Salgado and A. F. Rubio-Guerra, "Diabetic nephropathy and inflammation," World Journal of Diabetes, vol. 5, no. 3, pp. 393-398, 2014.

[44] Y. Zhou, L. Fang, L. Jiang et al., "Uric acid induces renal inflammation via activating tubular NF- $\kappa \mathrm{B}$ signaling pathway," PLoS One, vol. 7, no. 6, article e39738, 2012.

[45] J. Xiao, C. Fu, X. Zhang et al., "Soluble monosodium urate, but not its crystal, induces toll like receptor 4-dependent immune activation in renal mesangial cells," Molecular Immunology, vol. 66, no. 2, pp. 310-318, 2015.

[46] M. B. Sangoi, J. A. M. de Carvalho, E. Tatsch et al., "Urinary inflammatory cytokines as indicators of kidney damage in type 2 diabetic patients," Clinica Chimica Acta, vol. 460, pp. 178183, 2016.

[47] S. Jones, S. Jones, and A. O. Phillips, "Regulation of renal proximal tubular epithelial cell hyaluronan generation: implications for diabetic nephropathy," Kidney International, vol. 59, no. 5, pp. 1739-1749, 2001.

[48] P. Ranganathan, C. Jayakumar, and G. Ramesh, "Proximal tubule-specific overexpression of netrin-1 suppresses acute kidney injury-induced interstitial fibrosis and glomerulosclerosis through suppression of IL-6/STAT3 signaling," American Journal of Physiology-Renal Physiology, vol. 304, no. 8, pp. F1054-F1065, 2013.

[49] J. F. Navarro-González, C. Mora-Fernández, M. M. de Fuentes, and J. García-Pérez, "Inflammatory molecules and pathways in the pathogenesis of diabetic nephropathy," Nature Reviews Nephrology, vol. 7, no. 6, pp. 327-340, 2011. 


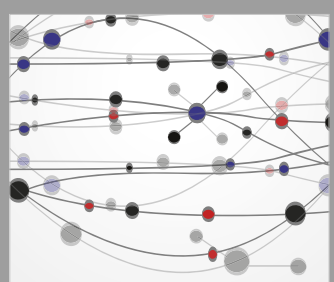

The Scientific World Journal
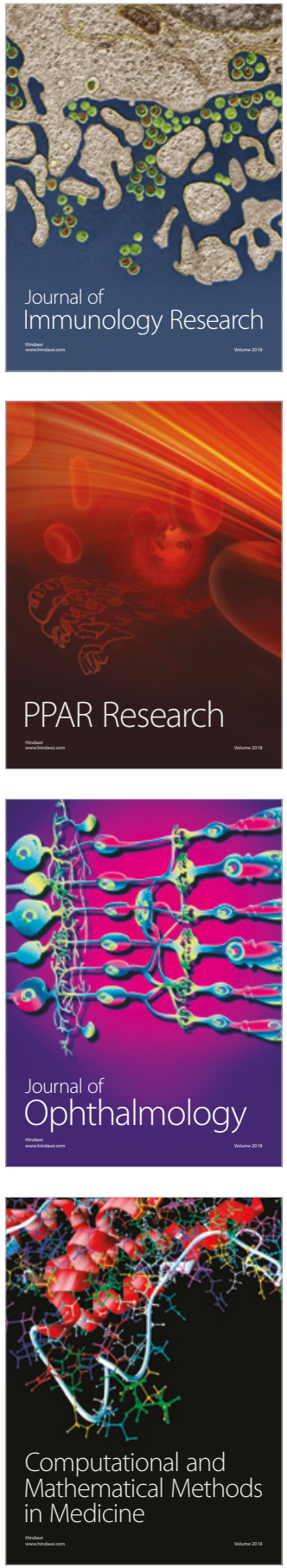

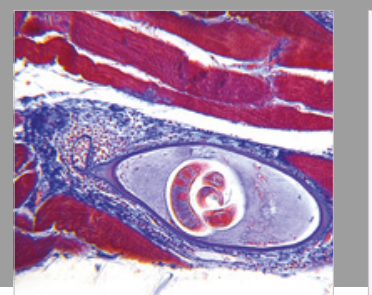

Gastroenterology Research and Practice

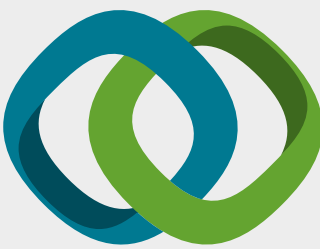

\section{Hindawi}

Submit your manuscripts at

www.hindawi.com
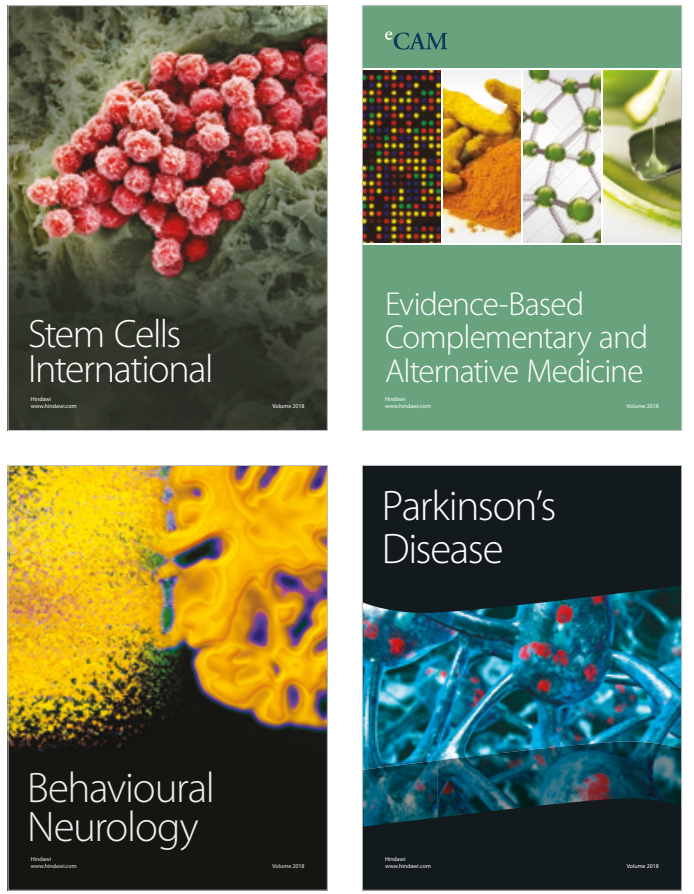

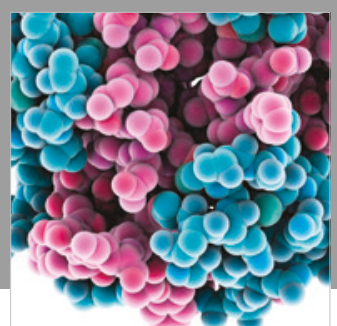

ournal of

Diabetes Research

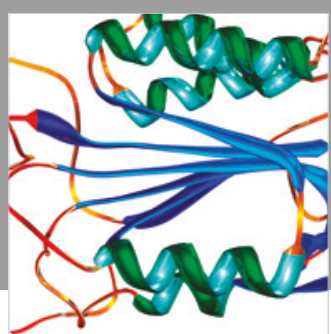

Disease Markers
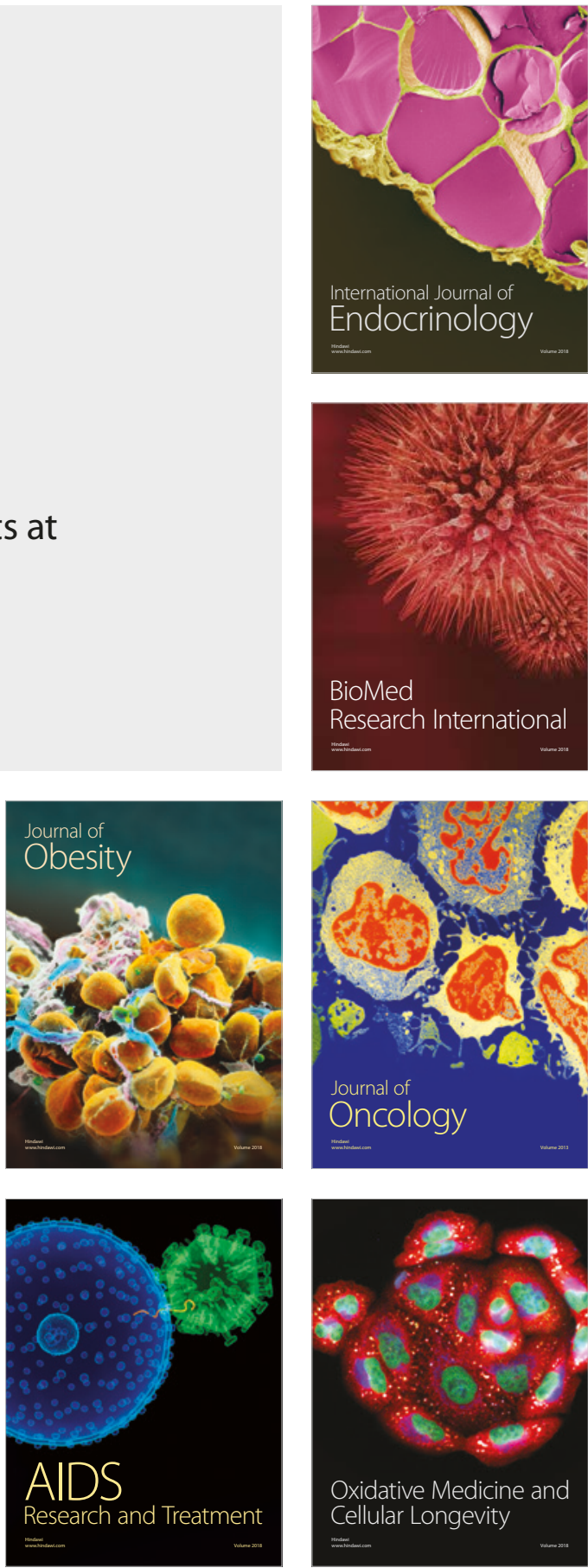